\title{
Práticas de manejo do Cateter Central de Inserção Periférica em uma unidade neonatal
}

\author{
Management practices of Peripherally Inserted Central Catheter at a neonatal unit
}

Prácticas de manejo del Catéter Central de Inserción Periférica en una unidad neonatal

\section{Eny Dórea', Talita Elci de Castro", Priscila Costa"', Amelia Fumiko Kimura"', Fernanda Matilde Gaspar dos Santos ${ }^{\mathrm{IV}}$}

'Universidade Federal Fluminense, Escola de Enfermagem Aurora de Afonso Costa, Departamento de Enfermagem Materno-Infantil e Psiquiátrica. Niterói-RJ. Universidade de São Paulo, Escola de Enfermagem,

Programa de Pós-Graduação em Enfermagem (Doutoranda). São Paulo-SP, Brasil.

" Hospital e Maternidade São Luiz, Unidade de Terapia Intensiva Neonatal. São Paulo-SP, Brasil.

III Universidade de São Paulo, Escola de Enfermagem,

Departamento de Enfermagem Materno-Infantil e Psiquiátrica. São Paulo-SP, Brasil.

Iv Centro Universitário Monte Serrat, Curso de Graduação em Enfermagem. Santos-SP, Brasil.

\section{Submissão 02-09-2010 Aprovação: 03-02-2012}

\section{RESUMO}

O estudo objetivou descrever o manejo dos Cateteres Centrais de Inserção Periférica instalados em neonatos internados em uma unidade de cuidado intensivo neonatal de um hospital privado, após a adoção de protocolo institucional. Realizou-se um estudo quantitativo descritivo-exploratório com coleta de dados prospectiva. Foram analisados dados de instalação de 45 cateteres. A remoção eletiva ocorreu em $63,8 \%$ dos cateteres e ocorreram complicações em 30,8\% dos cateteres. As complicações mais frequentes foram ruptura $(15,4 \%)$, oclusão $(11 \%)$ e tração acidental $(4,4 \%)$. Em $33 \%$ dos cateteres instalados não havia registro sobre curativo. Conclui-se que as complicações estiveram relacionadas ao cuidado do cateter, requerendo a capacitação dos profissionais e novos instrumentos para registro da assistência clínica de enfermagem em relação ao manejo do cateter.

Descritores: Enfermagem; Enfermagem neonatal; Cateterismo venoso central; Avaliação em enfermagem.

ABSTRACT

The study aimed to describe the management of Peripherally Inserted Central Catheters insertion in neonates admitted at a neonatal intensive care unit of a private hospital, after implementation of the institutional protocol. An exploratory, quantitative descriptive study with prospective data collection was carried out. Data of 45 catheters insertion were analyzed. The rate of catheter's with elective removal was $63.8 \%$, and catheter's complications rate was $30.8 \%$. The most frequently complications were rupture $(15.4 \%)$, occlusion $(11 \%)$ and accidental dislodgement $(4.4 \%)$. No records about the catheter dressings were observed in $33 \%$ of catheter's insertion. One conclude that the complications were related to care practices of the catheter, requiring professional training and new tools to record the care provided in relation to the catheter's management.

Key words: Nursing; Neonatal nursing; Central venous catheterization; Nursing assessment.

\section{RESUMEN}

El estudio tuvo como objetivo describir el manejo de los Catéteres Centrales de Inserción Periférica instalados en los recién nacidos internados en una unidad neonatal de cuidados intensivos de un hospital privado, después de la adopción del protocolo institucional. Fue realizado un estudio cuantitativo, descriptivo y exploratorio con recolecta de datos prospectiva. Fueron analizados datos de 45 catéteres instalados. El retiro electivo ocurrió en el $63,8 \%$ de los catéteres instalados y complicaciones ocurrieron en $30,8 \%$ de los catéteres. Las complicaciones más frecuentes fueron rotura $(15,4 \%)$, oclusión $(11 \%)$ y tracción accidental $(4,4 \%)$. En $33 \%$ de los catéteres instalados no hubo registro de los curativos. Concluyese que las complicaciones estuvieron relacionadas con el cuidado del catéter, siendo necesaria la capacitación de los profesionales, así como nuevos instrumentos para registrar la asistencia clínica de enfermería relacionada al manejo del catéter.

Palabras clave: Enfermería; Enfermería neonatal; Cateterización venosa central; Evaluación de enfermería.

\section{AUTOR CORRESPONDENTE Eny Dorea E-mail: enydorea@ig.com.br}




\section{INTRODUÇÃO}

A manutenção de acesso venoso em neonatos clinicamente instáveis, internados em unidades neonatais, é um desafio aos profissionais responsáveis pela sua assistência. Algumas características da terapêutica intravenosa prescritas aos neonatos, dentre elas, alta osmolaridade e concentrações superiores a $12,5 \%$ são irritantes e vesicantes aos vasos periféricos e inviabilizam sua administração por cateter venoso periférico curto.

Na prática clínica, o uso do Cateter Central de Inserção Periférica (CCIP) expandiu-se nas últimas décadas, com base no desenvolvimento dos dispositivos vasculares e do conhecimento de suas indicações. Pela Resolução n 258/2001, o Conselho Federal de Enfermagem dispôs ser de atribuição do enfermeiro a inserção, manutenção e a remoção do CCIP, quando houver indicação de seu uso ${ }^{(1)}$.

O CCIP é um cateter longo e flexível com comprimento que varia de 8 a $75 \mathrm{~cm}$, inserido por veia periférica que progride através desta até o sistema venoso central, onde sua ponta deverá ser alocada no terço inferior da veia cava superior ou no terço superior da veia cava inferior ${ }^{(2)}$.

Dois tipos de material de cateter são produzidos para a população de neonatos: o de poliuretano e o de silicone. Outra especificidade dos cateteres disponíveis no mercado é sua disponibilidade em lúmen único (monolúmen) e duplo (duplolúmen). O cateter de duplolúmen é apropriado a neonatos que recebam nutrição parenteral total, múltiplos medicamentos ou durante a reanimação ${ }^{(3)}$.

O CCIP é indicado para terapêutica parenteral prolongada e a vantagem de seu uso é ser mais seguro para infusão de soluções vesicantes, causando menor estresse, desconforto e redução da frequência de exposição à punção venosa, além do menor custo em relação ao cateter venoso central inserido cirurgicamente. $\mathrm{O}$ cateter venoso central (CVC) tuneilizado ou cirurgicamente inserido é associado a maior taxa de infecção comparado ao $\mathrm{CCIP}^{(3)}$.

As desvantagens do uso do CCIP estão relacionadas à necessidade de profissionais habilitados para sua inserção, de uma rede vascular calibrosa e íntegra e disponibilidade de equipamento de radiografia para verificação da localização anatômica de sua ponta, além de monitorização rigorosa do dispositivo quanto às complicações decorrentes de seu uso,

A prevalência de complicações relacionadas ao uso do CCIP, como oclusão, extravasamento, migração da ponta e trombose, varia entre $15 \%$ e $48 \%{ }^{(4)}$, o que reduz o tempo de permanência do dispositivo, o sucesso na implementação da terapêutica medicamentosa e a sobrevida dos neonatos.

Durante a instalação e manutenção do CCIP, as práticas de enfermagem adquirem importância como estratégia de segurança ao paciente por influenciarem na ocorrência de complicações e consequente remoção não eletiva.

Os serviços de saúde têm se organizado e vêm criando grupos de estudos voltados a discutir, propor e aprimorar os protocolos de procedimentos invasivos, incluindo o uso de dispositivos intravasculares. A padronização dos procedimentos de enfermagem, as estratégias de discussão, treinamento e a implementação de rotinas para utilização do CCIP podem contribuir para a redução de complicações relacionadas ao uso do cateter ${ }^{(5)}$

A finalidade deste estudo foi caracterizar as práticas implementadas no manejo dos CCIPs instalados em neonatos internados em uma Unidade de Terapia Intensiva Neonatal de um hospital privado da cidade de São Paulo, após a adoção de protocolo de inserção, manutenção e remoção do CCIP, com vistas à proposição de programas de educação contínua no manejo desse dispositivo.

Objetiva-se também identificar os sítios de inserção para instalação do CCIP, a frequência de punções venosas realizadas e o tempo de uso do dispositivo, além das indicações e motivos das remoções não eletivas do CCIP.

\section{METODOLOGIA}

Estudo quantitativo descritivo-exploratório cujos dados foram obtidos de observações e registros de dados de prontuários de neonatos internados na Unidade de Terapia Intensiva Neonatal (UTIN) de um hospital privado de São Paulo, submetidos à instalação do CCIP no período de 1 a 21 de agosto de 2009. Esta UTIN dispõe de 56 leitos, e o quadro funcional de enfermeiros é composto por 24 profissionais, dos quais são 18 habilitados em procedimento de inserção, manutenção e remoção do CCIP.

O manejo do CCIP na instituição do estudo segue as diretrizes definidas em um protocolo elaborado pelos enfermeiros do grupo de estudo em cateteres venosos em atividade, desde 2000. A indicação de inserção e remoção do CCIP é uma atribuição médica. Os tipos de cateteres utilizados na instituição são o de duplolúmen, de poliuretano com 2 Frenchs, indicado para infusão de nutrição parenteral total (NPT) e antibióticos; e o de monolúmen, de silicone e 1,9 Frenchs, indicado aos neonatos com prescrição de um único tipo de solução intravenosa. A inserção do CCIP é um procedimento asséptico realizado à beira do leito.

Sua manutenção efetiva-se com o procedimento de permeabilização periódica do cateter, visando a evitar a obstrução. O procedimento consiste em aspirar o cateter e, em seguida, infundir $1 \mathrm{ml}$ de solução fisiológica $0,9 \%$ em intervalos de 6 horas, ou infundir solução fisiológica 0,9\% imediatamente antes e após a administração de medicamentos. Vale ressaltar que é recomendado o uso de seringas de $10 \mathrm{ml}$ para permeabilizar o cateter, pois seringas de menor volume exercem maior pressão intravascular, aumentando a ocorrência de extravasamentos e perda de acesso venoso. Aos profissionais é recomendada a adoção da prática de assepsia do canhão (hub) do CCIP com solução alcoólica 70\%, manipulando-o com luvas estéreis. O cateter é liberado para uso, quando se constata, por imagem radiográfica, que sua ponta está alocada no terço inferior de veia cava superior, quando inserido nas veias dos membros superiores ou da região cefálica; ou no terço superior de veia cava inferior, se inserido por veias de extremidades inferiores.

O material a ser utilizado no curativo depende do peso do recém-nascido (RN), em função da fragilidade da pele. Em neonatos com peso inferior a 1.500 gramas, o antisséptico usado 
no local de inserção do cateter é clorexidine degermante; e a solução fisiológica $0,9 \%$ é empregada para a remoção do antisséptico. Nos RNs com peso igual ou superior a 1.500 gramas, o antisséptico indicado é a solução de clorexidine alcoólica; e a solução fisiológica 0,9\% é empregada para remover o excesso de clorexidine alcoólica. O cateter é fixo com a técnica de sutura de pele utilizando adesivo, em seguida é ocluído com película transparente, que consiste em uma membrana semipermeável que protege a entrada de micro-organismos e possibilita visualizar o aspecto do local de inserção do CCIP. A troca do curativo é realizada com técnica asséptica, quando a película transparente perder sua aderência ou com intervalo máximo de sete dias.

A indicação de remoção do CCIP é atribuição médica, mas a remoção é realizada pelo enfermeiro. Na ocorrência de sinais indicativos de infecção (hiperemia local, calor, edema e secreção purulenta no sítio de inserção do CCIP), é solicitado exame de cultura da ponta do CCIP. O enfermeiro registra a remoção do dispositivo CCIP e os achados observados no prontuário.

Neste estudo, os dados foram registrados em instrumento específico composto de duas partes. A primeira referia-se à caracterização do RN: sexo, peso na data de inserção do CCIP, diagnóstico principal, idade gestacional e idade pós-natal.

Na segunda parte do instrumento de coleta de dados, as informações relativas ao manejo do CCIP e ocorrências durante a permanência do dispositivo foram registradas. As seguintes informações foram coletadas:

$\checkmark$ Dados da inserção: tipo de cateter, utilização de analgésico ou sedativo, número de tentativas de punção, membro e veia puncionada, horário de início e término do procedimento, localização anatômica da ponta do cateter após imagem radiológica e solução infundida após a liberação de seu uso;

$\checkmark$ Dados de manutenção: motivo de realização do curativo, solução antisséptica usada e tipos de complicações relacionadas ao uso do cateter; e

$\checkmark$ Dados da remoção: motivo de remoção (eletiva ou não eletiva), encaminhamento da ponta para cultura (sim ou não), tempo de permanência do cateter e intercorrências durante a remoção.

Definição de termos

$\checkmark$ Oclusão: quando não há refluxo da solução infundida ou de sangue à aspiração do cateter com seringa de $10 \mathrm{~mL}$, ou impedimento total ao se infundir solução fisiológica $0,9 \%$, ou de medicamento pelo cateter;

$\checkmark$ Ruptura: falha na solução de continuidade em qualquer parte do cateter, confirmada pelo extravasamento da solução infundida pelo local da ruptura;

$\checkmark$ Infecção (ou suspeita de) relacionada a cateter: ocorrência de sinais clínicos indicativos de infecção: febre, secreção purulenta no local de inserção do cateter, piora clínica importante sem outro foco de infecção, resultado de hemocultura central ou cultura de ponta de cateter com crescimento maior ou igual a 15 unidades formadoras de colônia com técnica semiquantitativa; e

$\checkmark$ Remoção acidental do CCIP: exteriorização não intencional do cateter com subsequente desalojamento de sua ponta.

O estudo foi aprovado pelo Comitê de Ética em Pesquisa da Instituição, campo do estudo (Processo CEP 219/09). Os colaboradores envolvidos (enfermeiros responsáveis pela instalação do cateter CCIP) assinaram o Termo de Consentimento Livre Esclarecido para ciência da leitura de todas as anotações realizadas por eles durante a assistência de enfermagem, sem sofrer qualquer prejuízo.

Os dados foram armazenados em planilha eletrônica no aplicativo Microsoft Excel, e os resultados foram apresentados em tabelas e gráficos, com frequência absoluta e relativa.

\section{RESULTADOS}

Dados referentes ao manejo do CCIP de 45 recém-nascidos que fizeram uso desse dispositivo foram analisados. A maioria era do sexo masculino, $33(73,3 \%)$ tinham idade gestacional maior que 30 semanas $(62,2 \%)$, peso inferior a 2.000 gramas $(62,2 \%)$, com diagnóstico de afecção respiratória $(75,5 \%)$ e idade pós-natal na data de inserção do CCIP de 3 dias de vida $(53,3 \%)$, conforme mostram os dados da Tabela 1 .

\section{Tabela 1. Perfil dos neonatos e tipo de CCIP inserido.} São Paulo-SP, 2009.

\begin{tabular}{|c|c|c|}
\hline Perfil dos neonatos e tipo de CCIP inserido & $\mathbf{N}$ & $\%$ \\
\hline \multicolumn{3}{|l|}{ Sexo } \\
\hline Feminino & 12 & 26,7 \\
\hline Masculino & 33 & 73,3 \\
\hline \multicolumn{3}{|l|}{ Idade gestacional ao nascimento } \\
\hline$<30$ sem & 17 & 37,8 \\
\hline $30^{1 / 7}$ a $35 \mathrm{sem}$ & 16 & 35,5 \\
\hline$\geq 35^{1 / 7} \mathrm{sem}$ & 12 & 26,7 \\
\hline \multicolumn{3}{|l|}{ Peso } \\
\hline$<1.000 \mathrm{~g}$ & 02 & 4,4 \\
\hline 1.000 a $1.500 \mathrm{~g}$ & 16 & 35,5 \\
\hline 1.500 a $2.000 \mathrm{~g}$ & 10 & 22,3 \\
\hline$>2.000 \mathrm{~g}$ & 17 & 37,8 \\
\hline \multicolumn{3}{|l|}{ Diagnóstico principal } \\
\hline Afecção respiratória & 34 & 75,5 \\
\hline Afecção gastrointestinal & 09 & 20,0 \\
\hline Sepse & 07 & 15,5 \\
\hline \multicolumn{3}{|l|}{ Idade pós-natal na data da inserção do cateter } \\
\hline$\leq 3$ dias & 24 & 53,3 \\
\hline 4 a 7 dias & 11 & 24,4 \\
\hline$\geq 7$ dias & 10 & 22,3 \\
\hline \multicolumn{3}{|l|}{ Tipo de cateter inserido } \\
\hline Monolúmen de silicone & 27 & 60,0 \\
\hline Duplolúmen de poliuretano & 18 & 40,0 \\
\hline
\end{tabular}

Fonte: Arquivo pessoal dos autores 
Nenhum neonato recebeu analgésico para ser submetido à inserção do CCIP e 9,7\% receberam sedativo.

\section{Tabela 2. Variáveis relacionadas ao sítio e veia de inserção, frequência de punção venosa, localização anatômica da ponta do cateter e tempo de permanência do CCIP. São Paulo-SP, 2009.}

\begin{tabular}{|c|c|c|}
\hline $\begin{array}{l}\text { Variáveis relacionadas à instalação } \\
\text { do cateter CCIP }\end{array}$ & $\mathbf{N}$ & $\%$ \\
\hline \multicolumn{3}{|l|}{ Membro puncionado } \\
\hline MSD & 23 & 51,1 \\
\hline MSE & 11 & 24,4 \\
\hline MID & 3 & 6,7 \\
\hline Outros & 8 & 17,8 \\
\hline \multicolumn{3}{|l|}{ Veia de acesso } \\
\hline Basílica & 10 & 22,0 \\
\hline Cefálica & 9 & 20,0 \\
\hline Axilar & 6 & 13,3 \\
\hline Outras & 20 & 44,7 \\
\hline \multicolumn{3}{|l|}{ Frequência de punções } \\
\hline$\leq 3$ & 32 & 71,1 \\
\hline 4 a 6 & 6 & 13,3 \\
\hline$\geq 7$ & 7 & 15,6 \\
\hline \multicolumn{3}{|l|}{ Localização da ponta } \\
\hline Central & 34 & 75,6 \\
\hline Não central & 9 & 20,0 \\
\hline Sem registro & 2 & 4,4 \\
\hline \multicolumn{3}{|l|}{ Tempo de permanência do CCIP } \\
\hline$>5$ dias & 14 & 28,8 \\
\hline 5 a 10 dias & 17 & 37,7 \\
\hline$>10$ dias & 15 & 33,3 \\
\hline
\end{tabular}

Fonte: Arquivo pessoal dos autores

A média do tempo para a instalação do CCIP nos neonatos foi de 38,44 minutos, com tempo mínimo de 5 minutos e máximo de 120 minutos. As soluções infundidas pelo CCIP foram: nutrição parenteral prolongada e antibióticos, $25(55,5 \%)$; soro e antibióticos, $9(20,0 \%)$ e exclusivamente antibióticos, 6 (13,3\%). Os curativos durante a permanência do cateter foram realizados por indicação eletiva conforme descrito no protocolo institucional. Solução de clorexidine e soro fisiológico 0,9\% foram usados em $14(31,1 \%)$ neonatos, solução alcoólica e soro fisiológico 0,9\% em 8 (17,8\%) neonatos e, exclusivamente, o soro fisiológico em 4 (8,9\%) neonatos e solução de clorexidine degermante e soro fisiológico $0,9 \%$ em 4 (8,9\%) neonatos. Não foram identificados registros de curativo nos prontuários de 15 (33,4\%) neonatos com CCIP instalados. As remoções não eletivas de CCIP ocorreram em $16(35,2 \%)$ cateteres instalados.

Tabela 3. Indicação e motivos de remoção do CCIP. São Paulo-SP, 2009.

\begin{tabular}{lccc}
\hline \multicolumn{2}{l}{ Indicação e motivos de remoção } & N & \% \\
\hline Não eletiva & Ruptura & 14 & 30,8 \\
& Oclusão & 7 & 15,4 \\
& Remoção acidental & 5 & 11,0 \\
Eletiva & 2 & 4,4 \\
\hline & 29 & 63,8 \\
\hline & Óbito neonatal & 29 & 63,8 \\
\hline
\end{tabular}

Fonte: Arquivo pessoal dos autores

A ruptura do CCIP foi a ocorrência que mais motivou sua remoção não eletiva na população estudada, 7 (15,4\%) seguidas da oclusão, 5 (11,0\%). Os motivos de remoção estiveram relacionados à manutenção e não houve nenhuma complicação identificada na inserção nem em sua remoção.

A média do tempo de permanência do CCIP entre os neonatos estudados foi de 9,42 dias, variando de 1a 41 dias. Vale ressaltar que $29,5 \%$ dos cateteres permaneceram inseridos, por menos de 5 dias, 37,5\% mantiveram-se entre 5 e 10 dias, e 33,0\%, por mais de 10 dias. Nenhuma ponta de CCIP foi enviada para cultura por suspeita de infecção.

\section{DISCUSSÃO}

Os recém-nascidos submetidos à inserção de CCIP são, em sua maioria, constituídos de prematuros, de baixo peso e que necessitam desse dispositivo para garantir a implementação da terapêutica medicamentosa e, dessa forma, aumentar suas chances de sobrevida e melhor prognóstico.

Em virtude da maior prematuridade e gravidade do quadro clínico, nesse estudo 44,5\% dos CCIPs foram inseridos em RNs com menos de 48 horas de vida. Observa-se mudança na prática assistencial referente à utilização de CCIP. Antes, o procedimento de inserção do cateter era preferencialmente realizado a partir de 48 horas de vida do $\mathrm{RN}$ em razão da diminuição do edema de nascimento e melhora da visualização da rede venosa com aumento das chances de sucesso na inserção ${ }^{(6)}$.

A antecipação do procedimento de inserção demonstra a capacidade técnica da equipe de enfermeiros habilitada que compõe a unidade neonatal, já que esse procedimento é um processo de alta complexidade técnica e exige conhecimento específico ${ }^{(6)}$.

No momento do procedimento, a manutenção do $\mathrm{RN}$ calmo e confortável aumenta o sucesso na inserção e localização central do $\operatorname{CCIP}^{(7)}$. Nesse estudo, não houve uso de analgesia para inserção do CCIP e o emprego de sedativos ocorreu em 9,7\% dos procedimentos, figurando apenas como forma de redução da agitação do RN. O dado corrobora os resultados de investigação descritiva realizada com 24 
neonatos com objetivo de avaliar a resposta dolorosa durante inserção do CCIP apontando para a necessidade de se adotar medidas analgésicas, a exemplo da contenção, administração via oral de soluções adocicadas, sucção não nutritiva e também utilizando analgesia medicamentosa, uma vez que neonatos internados em unidade neonatal são, com frequência submetidos a procedimentos invasivos e dolorosos ${ }^{(8)}$.

A escolha da veia a ser puncionada é de extrema relevância no sucesso da inserção do cateter. Nesse estudo, a veia com maior frequência de acesso para inserir o CCIP foi a basílica, $22 \%$, seguida da veia cefálica, $20 \%$. As veias cefálica e a basílica são as mais utilizadas, sendo a basílica, a mais recomendada em razão de seu maior calibre, menor número de válvulas e a de mais fácil manipulação no procedimento de troca de curativo ${ }^{(6,8)}$.

Quanto ao sucesso na realização do procedimento, a taxa de sucesso até a terceira tentativa de punção foi $71,2 \%$, frequência semelhante à descrita em um estudo transversal prospectivo brasileiro realizado com 37 neonatos de uma unidade neonatal referência para gestação de alto risco cuja prevalência foi de $73,4 \%$. Este dado demonstra habilidade técnica da equipe de enfermeiros responsável pela inserção do dispositivo ${ }^{(9)}$.

Verificou-se ainda que em $75,5 \%$ dos cateteres inseridos, a ponta foi alojada centralmente, atendendo ao protocolo institucional, frequência pouco inferior ao descrito na literatura, de $83,3 \%{ }^{(9)}$. Um estudo descritivo do tipo survey analisou respostas de questionários enviados a 98 unidades de terapia intensiva neonatal do Japão com questões relacionadas às normas e protocolos institucionais sobre o manejo do CCIP. Identificou-se maior indicação de alocação da ponta do CCIP nas veias subclávia, cava superior, cava inferior e ilíaca externa. Apenas $9 \%$ admitiram manter a ponta do CCIP alocada em átrio direito. Aproximadamente um terço das instituições usou o CCIP midline ${ }^{(10)}$.

Embora o serviço adote um protocolo para o manejo do CCIP, os achados mostraram emprego de diferentes soluções antissépticas no curativo do CCIP. Observou-se que 15 $(33 \%)$ dos prontuários não continham registro sobre curativo e em apenas 17 dos casos (38\%) foram utilizadas as soluções definidas em protocolo institucional.

O papel do enfermeiro é de fundamental importância na realização dos cuidados do CCIP, conforme protocolo da unidade onde trabalha ${ }^{(6)}$. A elaboração de um impresso de fácil utilização pode ser uma forma de solucionar os problemas oriundos da falta ou inadequado registro de qualquer procedimento realizado com o CCIP, além do treinamento e utilização de indicadores que possam avaliar a assistência prestada.

A ocorrência de complicações nesse estudo, 30,8\%, foram similares aos dados da literatura que mostram que a prevalência de complicações mecânicas e infecciosas relacionadas ao uso do PICC em neonatos de baixo peso internados em UTIN varia de $20,7 \%$ a $62,2 \%(7,11)$. No Brasil, o estudo realizado na cidade de Santos avaliando 135 cateteres PICC demonstrou uma prevalência de complicações mecânicas e infecciosas de $62,2 \%{ }^{(11)}$. Estudo de coorte com 477 neonatos que objetivou comparar as taxas de complicação relacionadas ao CCIP inseridos em membros superiores e inferiores para infusão de nutrição parenteral, a taxa de complicações relacionada ao uso do dispositivo foi $30,7 \%{ }^{(12)}$.

A obstrução do CCIP é uma das complicações mais frequentes. Neste estudo, $5(11 \%)$ dos cateteres foram removidos por obstrução, em estudo nacional realizado com 14 RNs em UTIN de São Paulo, a incidência dessa complicação foi de $25 \%^{(13)}$. A principal estratégia de prevenção é a permeabilização meticulosa do cateter.

A ruptura do cateter pode ser por manuseio inadequado do dispositivo, especialmente nos cateteres de silicone. As possíveis causas da ruptura incluem o excesso de pressão no flush, utilizando seringa de menor volume, visto que o dispositivo não suporta pressões, quando se usa seringa com capacidade menor que $10 \mathrm{~mL}^{(3)}$. Outros fatores que podem influenciar são as mudanças na composição da equipe de enfermagem e profissionais em fase de treinamento.

A taxa de ruptura $15,4 \%$ verificada no presente estudo foi superior a estudo nacional, em que a ruptura ou perfuração do canhão motivou a remoção de $5,2 \%$ dos cateteres ${ }^{(11)}$.

A infecção relacionada ao cateter é frequente e há inúmeras definições e diferentes métodos de tratamento relatados na literatura ${ }^{(7)}$. Contaminações do hub, do lúmen do cateter ou da solução infundida são responsáveis pela maioria das infecções relacionadas ao cateter ${ }^{(14)}$. No presente estudo não houve nenhum caso de infecção relacionada ao CCIP, embora haja descrição na literatura da ocorrência em $2 \%$ a $3 \%$ dos $\mathrm{CCIPs}^{(15)}$.

A maioria das indicações de remoção foi eletiva, $63,8 \%$, ou seja, por término da terapia intravenosa, outros $4,4 \%$ foram removidos por óbito do neonato. Em estudo prospectivo que coletou dados de 185 cateteres instalados em neonatos internados em uma UTI neonatal, no período de 1998 a 2005 , a taxa de remoção eletiva foi $65 \%$.

A maioria dos cateteres $(70,5 \%)$ permaneceu por mais de 5 dias, semelhante a estudos internacionais que mostram que a média de permanência do PICC foi de 12,2 dias, variando de 1 a $38 \operatorname{dias}^{(7)}$.

\section{CONSIDERAÇÕES FINAIS}

As complicações relacionadas ao uso do CCIP ocorreram em taxas similares às relatadas na literatura, permitindo a implementação da terapêutica intravenosa na maioria dos neonatos. No entanto, os procedimentos de manutenção mostraram diversidade entre a prática assistencial e o protocolo institucional, sendo responsáveis pela ocorrência de algumas complicações.

Visto que a prática clínica demonstrou haver inconsistência em relação ao protocolo do CCIP adotado na unidade, é notória a importância da necessidade de investimento na capacitação dos profissionais da equipe de enfermagem e do desenvolvimento de instrumentos que direcionem o registro da assistência prestada. 


\section{REFERÊNCIAS}

1. Conselho Federal de Enfermagem (COFEN). Resolução COFEN- 258/2001 Inserção de Cateter Periférico Central pelos Enfermeiros. [Legislação na Internet]. São Paulo; 2001[citado 2011 jan 24]. Disponível em: http://site.portalcofen.gov.br/node/4296.

2. Freitas LCM. Curso de qualificação em inserção, utilização e cuidados com cateter Venoso Central de Inserção Periférica- Neonatologia e pediatria [CD-ROM]. São Paulo: SOBETI; 2004.

3. Pettit J, Wyckoff MM. Peripherally inserted central catheters: guideline for practice. $2^{\text {nd }}$ ed. Glenview: National Association of Neonatal Nurses, [diretriz na internet]. 2007 [cited 2011 Jan 15]. Available from: http://www.nann.org/ pdf/PICCGuidelines.pdf.

4. Shah PS, Shah VS. Continuous heparin infusion to prevent thrombosis and catheter occlusion in neonates with peripherally placed percutaneous central venous catheters. Cochrane Database of Syst Rev. 2009;16(2):CD002772.

5. Menezes SO. Avaliação do acesso vascular em neonatos com menos de 1500g internados em Unidades Neonatais da SMS do Rio de Janeiro. [Dissertação]. Instituto Fernandes Figueira, Fundação Oswaldo Cruz. Rio de Janeiro; 2005.

6. Rodrigues ZS, Chaves EMC, Cardoso MVLML. Atuação do enfermeiro no cuidado com o cateter central de inserção periférica no recém-nascido. Rev Bras Enferm 2006;59(5):626-9.

7. Paulson PR, Miller KM. Neonatal Peripherally Inserted Central Catheters: recommendations for prevention of insertion and postinsertion complications. Neonatal Network 2008;27(4):245-57.
8. Costa P, Camargo PP, Bueno M, Kimura AF. Dimensionamento da dor durante a instalação do cateter central de inserção periférica em neonatos. Acta Paul Enferm 2010;23(1):35-40.

9. Camargo PP, Kimura AF, Toma E, Tsunechiro MA. Localização inicial da ponta de cateter central de inserção periférica (PICC) em recém-nascidos. Rev Esc Enferm USP 2008;42(4):723-38.

10. Ohki Y, Yoshizawa Y, Watanabe M, Kuwashima M, Morikawa A. Complications of percutaneously inserted central venous catheters in japanese neonates. Pediatrics International 2008;50(5):636-9.

11. Lourenço AS, Kakehashi TY. Avaliação da implantação do cateter central de inserção periférica em neonatologia. Acta Paul Enferm. 2003;16(2):26-32.

12. Hoang V, Sills JP, Chandler M, Busalani E, Clifton-Koeppel, Modanlou HD. Percutaneously Inserted Central Catheter for Total Parenteral Nutrition in Neonates: Complications Rates Related to Upper Versus Lower Extremity Insertion. Pediatrics 2008;121(5):1152-9.

13. Freitas $E M, N$ unes $A B$. O enfermeiro na práxis de cateter central de inserção periférica em neonato. Rev Min Enferm. 2009;13(2):215-24.

14. Garland JS, Alex CP, Sevallius JM, Murphy DM, Good MJ, Volberding AM, et al. Cohort study of the pathogenesis and molecular epidemiology of catheter-related bloodstream infection in neonates with peripherally inserted central venous catheters. Infect Control Hosp Epidemiol. 2008;29(3):243-9.

15. Jesus VC, Secoli SR. Complicações acerca do cateter venoso central de inserção periférica (PICC). Cienc Cuid Saúde 2007;6(2):252-60. 